\title{
Desarrollo de una base de datos para el estudio de los aditivos alimentarios en las asignaturas de química de alimentos
}

\section{Development of a database for the study of food additives in food chemistry subjects}

\author{
Juan Martín Gómez ${ }^{1}$, María Lourdes Moyano Cañete², Luis Zea Calero³, Julieta \\ Mérida $^{4}$ \& María Pérez Serratosa ${ }^{5}$
}

Fecha de recepción: 05/10/2020; Fecha de revisión: 27/10/2020; Fecha de aceptación: 11/02/2021

Cómo citar este artículo:

Martín Gómez, J., Moyano-Cañete, M.L., Zea-Calero, L., Mérida-García, J., \& Pérez-Serratosa, M. (2021). Desarrollo de una base de datos para el estudio de los aditivos alimentarios en las asignaturas de química de alimentos. Revista de Innovación y Buenas Prácticas Docentes, 10(1), 109-118.

Autor de Correspondencia: juanmartingomez@outlook.es

\begin{abstract}
Resumen:
Las TIC o tecnologías de la información y comunicación son una herramienta que permite un mayor desarrollo personal para el alumno, siendo él el protagonista en lugar del profesor. Con estas herramientas se adquieren diferentes competencias básicas necesarias para los alumnos universitarios, como son la capacidad de trabajo en equipo, la creatividad, el aprendizaje autónomo, entre otras. Además, los alumnos fueron partícipes en la evaluación de las actividades fomentando así también las competencias básicas relacionadas con la evaluación, como la reflexión.

El objetivo de este trabajo era desarrollar una base de datos sobre aditivos, incluyendo información sobre clasificación, fórmula química, grupo al que pertenece, masa molecular, toxicidad, ingesta diaria admitida. La base de datos se crea mediante la participación de alumnos en diferentes actividades de búsqueda de información. Esta base de datos estaría disponible para los alumnos y serviría a su vez al profesor para la realización de nuevas actividades a través de la plataforma Moodle de la Universidad de Córdoba.
\end{abstract}

Palabras clave: Aditivos, Base de datos, TIC, Competencias básicas

\begin{abstract}
:
ICT or information and communication technologies are a tool that allows a greater personal development for the student, being the protagonist instead of the teacher. With these tools, different basic competencies necessary for university students are acquired, such as teamwork, creativity, autonomous learning, among others. In addition, students were involved in the evaluation of activities, thus also promoting the basic competencies related to evaluation, such as reflection.

The objective of this job was to develop a database on additives, including information on classification, chemical formula, group to which it belongs, molecular mass, toxicity, acceptable daily intake. The database is created through the participation of students in different information search activities. This database would be available to students and would in turn serve the professor for the realization of new activities through the Moodle platform of the University of Córdoba.
\end{abstract}

Key Words: Additives, Database, ICT, Basic competencies

\footnotetext{
1 Universidad de Córdoba (España), juanmartingomez@outlook.es; CÓDIGO ORCID: 0000-0002-0791-7490

2 Universidad de Córdoba (España), qe1mocal@uco.es; CÓDIGO ORCID: 0000-0002-2319-4843

3 Universidad de Córdoba (España), qe1zecal@uco.es

${ }^{4}$ Universidad de Córdoba (España), qe1megaj@uco.es; CÓDIGO ORCID: 0000-0002-8137-7082

${ }^{5}$ Universidad de Córdoba (España), q72pesem@uco.es; CÓDIGO ORCID: 0000-0003-0244-947X
} 


\section{INTRODUCCIÓN}

Según el nuevo Espacio de Educación Superior el alumno debe emplear un mayor tiempo realizando un trabajo personal, siendo el protagonista en lugar del profesor. Por ello, es necesario el desarrollo de actividades diversificadas y atractivas que permitan un mayor desarrollo personal del alumno

En este sentido las Tecnologías de la Información y la Comunicación (TIC) se presentan como una herramienta adecuada y eficaz tanto para el alumno como para el profesor. El e-learning ha permitido el diseño de propuestas novedosas para enseñar, para compartir materiales instruccionales y para navegar a través de ellos de forma estructurada (Cataldi \& Lage, 2012).

El ámbito del e-learning y la gestión del conocimiento ligados al desarrollo de la educación y de la formación está en proceso de crecimiento y de desarrollo permanente. Un planteamiento educativo/formativo no tiene ningún sentido sin la inclusión de las TIC.

Este nuevo sistema de aprendizaje presenta algunas ventajas como instrumento de formación personal

- Eficaz combinación de estudio y trabajo

- Formación fuera del aula

- Permite una actualización continua adaptándose a los requerimientos de los alumnos

- El alumno puede marcar su ritmo de aprendizaje en cierta medida

- Existe una comunicación bidireccional, por lo que el aprendizaje es más dinámico

Para ello se propone utilizar las bases de datos como herramienta que permite recopilar gran cantidad de información y gestionarla posteriormente según los intereses de cada momento.

Un sistema de base de datos es básicamente un sistema computarizado cuya finalidad general es almacenar información y permitir a los usuarios recuperar y actualizar esa información por medio de consultas o peticiones perfectamente definidas. La información en cuestión puede ser cualquier cosa que sea de importancia para el individuo u organización; en otras palabras, todo lo que sea necesario para auxiliarle en el proceso de su administración.

Los Aditivos Alimentarios son un conjunto de compuestos muy amplio que, independientemente de su valor nutricional, son añadidos intencionadamente en los alimentos con fines tecnológicos (Ibáñez, Torre, \& Irigoyen, 2003). Su estudio descriptivo representa una parte muy importante del programa de la asignatura de Química Agrícola y Agroalimentaria. En todo caso, el aspecto descriptivo de los aditivos utilizados por la industria alimentaria se ajusta muy bien a los requisitos básicos de los sistemas multimedia, ya que es fácil agrupar sus características en tablas de datos cuya estructura es relativamente sencilla, pero, a la vez, ofrecen una gran versatilidad de uso. Toda la información acumulada será recuperada posteriormente por el alumno para poder realizar diferentes actividades en el estudio de los Aditivos utilizados en la Industria Alimentaria.

Por todo ello, se pretende mejorar el proceso de aprendizaje mediante la creación de una base de datos y el diseño de actividades que permitan a los alumnos indagar y realizar un estudio dinámico de los Aditivos usados en la Industria Alimentaria, realizando, de esta manera, un aprendizaje más activo. Además, los alumnos podrán evaluar la idoneidad de las actividades a realizar. 


\section{DESARROLLO DE LA EXPERIENCIA DE INNOVACIÓN}

\subsection{Creación de una plantilla para la recopilación de datos}

En primer lugar, se desarrolló una plantilla para la base de datos, donde se incluían los datos que se pretendían introducir en la base de datos.

Los campos que se querían incluir en la base de datos de los aditivos fueron:

- Código E: códigos asignados a los aditivos alimentarios en la Unión Europea

- Nombre del compuesto: nombre con el que se conoce al compuesto

- Nombre científico: según la nomenclatura de la IUPAC

- Fórmula molecular: representación convencional de los elementos que forman una molécula

- Número EINECS (European Inventory of Existing Chemical Substances): es un número de registro dado a cada sustancia química comercialmente disponible en la Unión Europea

- Descripción: en que formato se presenta o aspecto que tiene

- Masa molecular. En g/mol

- Grupo de aditivos al que pertenece: si son de protección, mejora, auxiliares tecnológicos o nutritivo

- Categoría: conservante, colorante, edulcorante...

- Toxicidad: en el caso que la presente

- Ingesta diaria admitida (IDA)

- Estructura química

\subsection{Creación de una base de datos}

Para este trabajo, la base de datos se creó mediante el programa Access de Microsoft, debido a que es uno de los programas más usados para este fin, y a que es el que se utiliza en los cursos de formación del profesorado impartidos por la Universidad de Córdoba.

La creación de la base de datos se realizó incluyendo en el programa Access toda la información obtenida de los aditivos por los alumnos.

En la información solicitada a los alumnos, se pedía la fórmula y estructura de los diferentes compuestos utilizados. Para obtener estas estructuras de forma homogénea se utilizó el programa ACD/ChemSketch Free Versión (5.12) que permite dibujar moléculas químicas, realizándolo para todos los compuestos estudiados. 


\subsection{Creación de una aplicación}

Finalizada la cumplimentación de la base de datos en el programa Access de Microsoft, se creó una aplicación llamada aditivos que permitiera manejar toda la información contenida en la base de datos de manera intuitiva y eficaz.

\subsection{Evaluación por el alumnado}

La actividad fue evaluada por los alumnos que participaron, de la asignatura de Química Agrícola y Agroalimentaria, en la creación de la base de datos. Para ello, se le puso a su disposición un cuestionario de calidad en la plataforma Moodle de la Universidad de Córdoba. Con ello, se pretendía evaluar de 1, la actividad no tiene carácter formativo, hasta 5 , la actividad ayuda a la comprensión del temario.

\section{RESULTADOS}

Con el presente trabajo se pretendía desarrollar una base de datos de los Aditivos Alimentarios que actualmente se utilizan en la Industria Alimentaria para el estudio de los mismos en las asignaturas de Química de Alimentos. La importancia de este trabajo es que permite elaborar una base de datos para que el alumno pueda encontrar con facilidad las características más importantes de los aditivos alimentarios a partir de su número de identificación europeo que aparece en la etiqueta del producto. Dar al alumno una visión general de los compuestos utilizados en la elaboración de los diferentes alimentos. Clasificar los aditivos presentes en las formulaciones de los alimentos según diferentes propiedades tales como código $\mathrm{E}$, naturaleza o propiedades fisicoquímicas. Vincular cada aditivo con su estructura química. Conocer el tipo de alimentos donde pueden encontrar cada uno de los compuestos estudiados. Proporcionar un método para que el alumno pueda reconocer rápidamente si una sustancia química es o no un aditivo alimentario autorizado, distinguiéndola de un posible ingrediente, contaminante o auxiliar tecnológico y facilitar al estudiante una visión amplia del mercado de los alimentos mediante la adquisición de un número considerable de ellos.

La legislación europea establece hasta 24 categorías de aditivos, aunque también se pueden clasificar en cuatro grandes grupos como son aditivos de conservación, aditivos de mejora, auxiliares tecnológicos y por último los aditivos nutritivos. La primera actividad que se planteó a los alumnos, en las sesiones de grupos medianos como parte del temario que incluyen las correspondientes asignaturas, consistía en la búsqueda e identificación de una serie de aditivos pertenecientes a diferentes categorías. Para ello se les entregó una ficha para rellenar con la información sobre los diferentes aditivos de una lista (Figura 1). 


\section{IdENTIFICACIÓN DE COMPUESTOS UTILIZAdos COMO EN Aditivos \\ en Etiquetas de Productos Comerciales}

\begin{aligned} & $\begin{array}{l}\text { Indicar de cada uno de los compuestos de la lista: } \\ \text { a) }\end{array}$ código E \\ & b) nombre del compuesto \\ & c) nombre científico \\ & d) fórmula molecular \\ & e) número EINECS \\ & f) descripción \\ & g) masa molecular \\ & h) categoría \\ & i) toxicidad \\ & j) ingesta diaria admitida (IDA) \\ & k) estructura química \\ & \\ & \hline 1 Acesulfamo-K \\ & \hline 2 Ácido Acético \\ & \hline 3 Anhídrido Sulfuroso \\ & \hline 4 Ácido ascórbico \\ & \hline 5 Curcumina \\ & \hline 6 Ácido málico \\ & \hline 7 Dióxido de Carbono \\ & \hline 8 Tocoferol \\ & \hline 9 Gelatina \\ & \hline 10 Aspartamo \\ & \hline 11 Glutamato Sódico \\ & \hline 12 Alginato \\ & \hline 13 Nisina \\ & \hline 14 Pectinas \\ & \hline 15 Sorbato Potásico \\ & \hline\end{aligned}

Figura 1. Modelo de ficha para la información sobre los aditivos.

En la figura 1 queda recogida la ficha modelo que se entregó a los alumnos para que buscaran información sobre los aditivos. Dicha ficha recoge la información que los alumnos debían de buscar sobre los aditivos y una lista de aditivos de las diferentes categorías. En la asignatura de Química Agrícola y Agroalimentaria había 86 alumnos matriculados y se hicieron grupos de 2 alumnos para desarrollar la actividad. Para que los alumnos pudieran llevar a cabo la actividad se les proporcionaron dos libros para usar como base de la información, aunque podían utilizar más herramientas a su alcance. Estos libros eran "Manual de utilización de aditivos en alimentos y bebidas" (Madrid, 1987) y "Aditivos y auxiliares de fabricación en las industrias agroalimentarias" (Multon, 1999) .

Posterior al desarrollo de la actividad, para que todos los alumnos aprendieran sobre los aditivos puestos en estudio, cada grupo preparó una presentación oral sobre un aditivo concreto y lo presentó ante sus compañeros, comentando incluso curiosidades sobre cada uno de ellos. 
A cada uno de los grupos se le solicitó la estructura de cada uno de los aditivos que tenían en estudio. Para dibujarlas hicieron uso del programa ACD/ChemSketch Free Versión (5.12). En la Figura 2 quedan recogidas las estructuras presentadas por un grupo a modo de ejemplo. Todas las estructuras presentadas se guardaron como imagen para poder incluir en el archivo como uno de los campos de la base de datos.

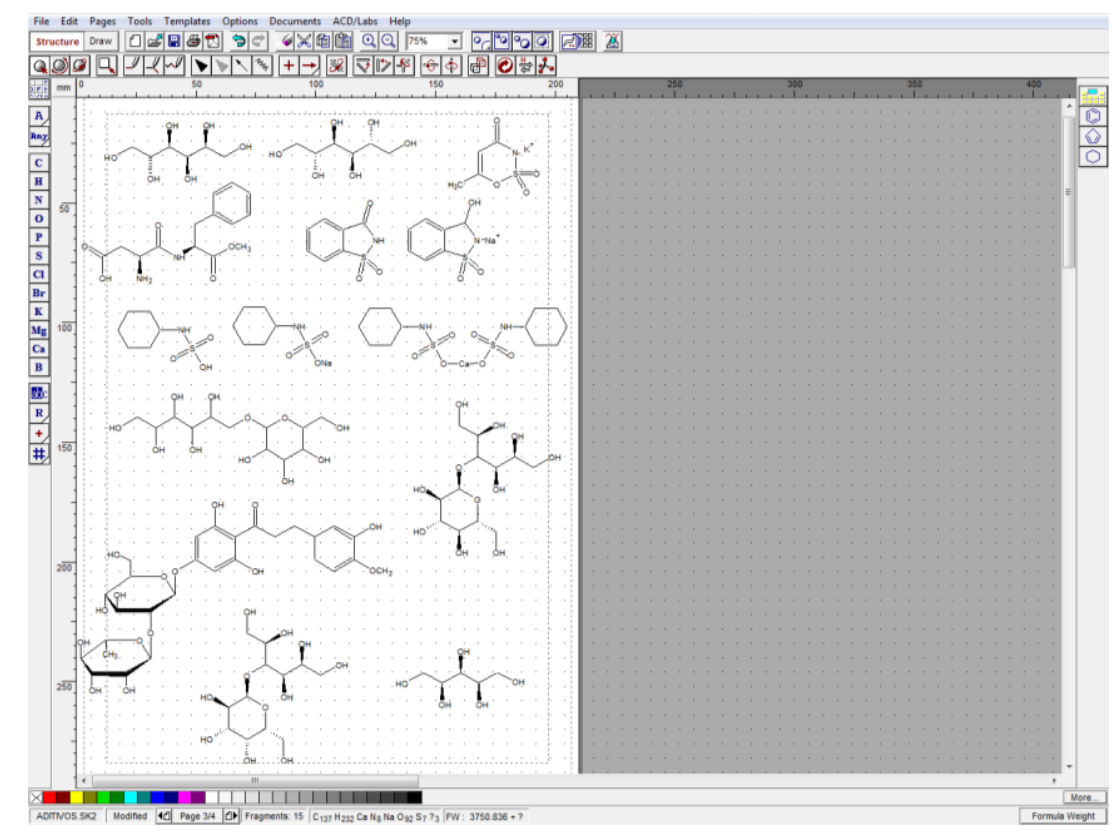

Figura 2. Creación de estructuras de los diferentes aditivos con el programa $\mathrm{ACD} /$ ChemSketch

Con esta actividad el docente adquiere los roles más significativos según diferentes autores, como son los de tutor, orientador, diseñador de situaciones mediadas de aprendizaje, evaluador, y creador de contenidos (Cabero-Almenara, 2010; CaberoAlmenara \& Gutiérrez Castillo, 2015; Llorente Cejudo, Gutiérrez Porlán, \& Barroso Osuna, 2015). Mientras que los alumnos adquieren diferentes competencias básicas de aprendizaje como son competencias instrumentales, en las que se engloban la capacidad de análisis y síntesis, de organización y planificación, y de gestión de la información; competencias personales como son la capacidad de trabajo en equipo; y por último competencias sistemáticas como el aprendizaje autónomo, la adaptación a nuevas situaciones, la creatividad y el liderazgo. Estas son algunas de las competencias genéricas que resumen algunos autores que deberían de adquirir los estudiantes en la docencia universitaria (González-Maura \& González-Tirados, 2008).

Una vez recopilada la información, se empezó a completar cada registro de la tabla de la base de datos incluyendo todos los apartados anteriores y las imágenes de todos los compuestos. En la Figura 3 se puede comprobar los registros de cada uno de los aditivos (filas) y los diferentes campos que se han ido completando para cada aditivo (columnas). 


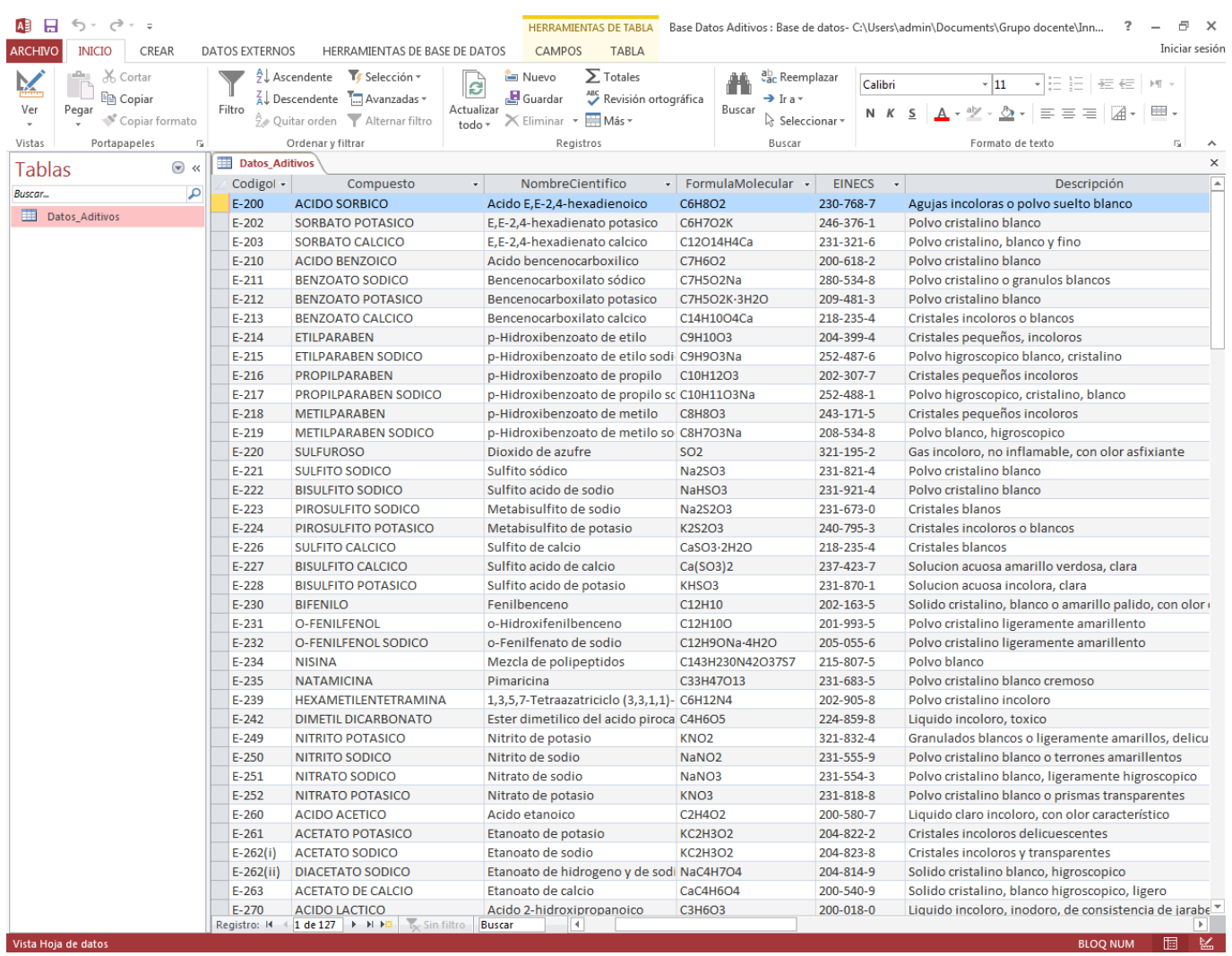

Figura 3. Base de datos de Aditivos.

Paralelamente a la base de datos se creó un programa que utilizaba la base de datos para la búsqueda de información que se denominó ADITIVOS. Este programa permite usar diferentes criterios de búsqueda. De esta forma, por ejemplo, se pueden buscar todos los aditivos que son ácidos (Figura 4) y se obtienen todos los compuestos en todas las categorías que son ácidos. Además, el programa también permite realizar nuevos registros sin utilizar el programa Access, lo que facilita el ir ampliando la base.

Por último, esta base de datos y el programa ADITIVOS se utilizarían para la realización de actividades dentro de la Plataforma Moodle, que tendrá como objetivos:

- Desarrollar una serie de actividades no presenciales, o dirigidas, que puedan ayudar al alumno a adquirir habilidades y destrezas en el ámbito de los aditivos alimentarios autorizados.

- Promover un aprendizaje virtual activo.

- Utilizar dichas actividades para la autoevaluación del alumno y la evaluación por parte del profesor de parte de la asignatura.

- Evaluar el grado de aceptación que estas actividades tienen por parte del alumnado.

- Garantizar que el alumno desarrolle algunas de las competencias exigidas para el desarrollo de su titulación. 


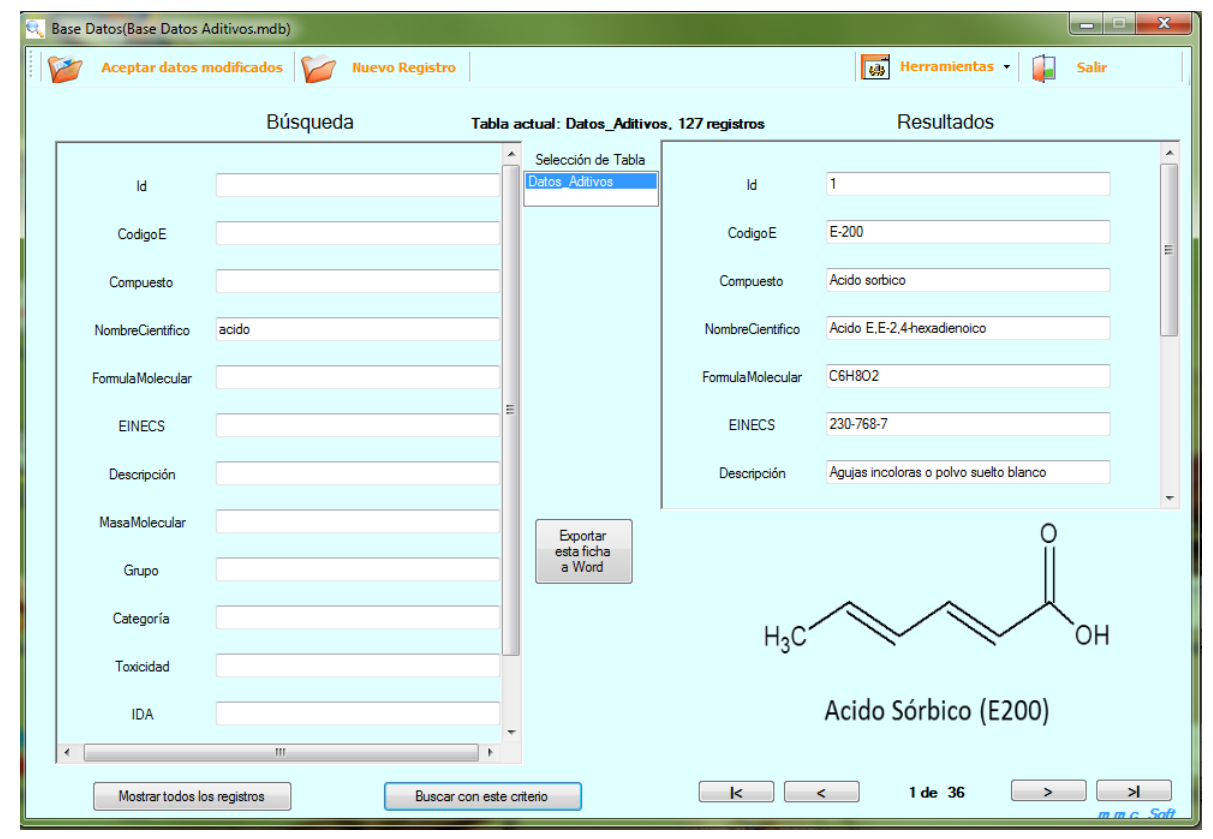

Figura 4. Programa ADITIVOS

Dentro de las competencias básicas que debe adquirir el alumnado universitario están las relacionadas con la evaluación. De acuerdo con los autores Rodríguez-Gómez et al. (2018) el alumnado potencia las competencias transversales cuando valoran sus actuaciones y producciones, las de sus compañeros e incluso cuando valoran al profesorado. Por este motivo, se puso a disposición de los alumnos un cuestionario en la plataforma Moodle para que evaluaran el carácter educativo de la actividad desarrollada, con la finalidad de que fomentaran su capacidad reflexiva. Para ello, los alumnos debían evaluar la actividad desde puntuaciones de 1, la actividad no tiene carácter formativo, hasta 5, la actividad ayuda a la comprensión del temario.

Tras el tiempo dispuesto para que el alumnado respondiera al cuestionario, un $67,4 \%$ de los alumnos matriculados en la asignatura de Química Agrícola y Agroalimentaria lo respondieron. En la figura 5 quedan resumidas las puntuaciones obtenidas de esta evaluación, donde el $47 \%$ del alumnado que los respondió pensaba que la actividad ayudaba a la comprensión del temario. Únicamente un $20 \%$ del alumnado la evaluó con una puntuación de un 3 o menor. Siendo la puntuación media obtenida un 4,2. Además, según comentaron les ayudó a tener una visión más real de la industria agroalimentaria y a ser más críticos con respecto al uso de determinados compuestos en los alimentos, tema que es muy actual hoy en día. 


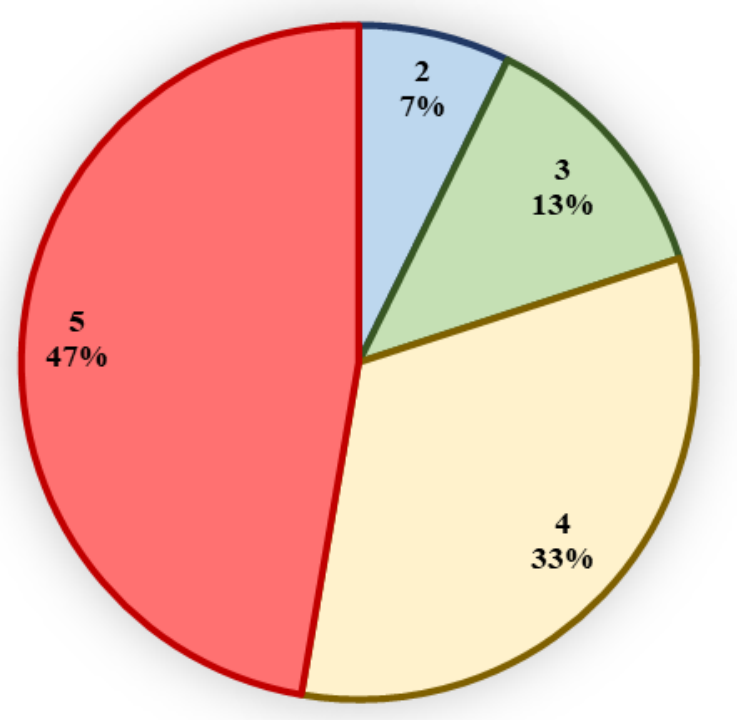

Figura 5. Evaluación de la actividad por el alumnado.

\section{CONCLUSIONES}

La actividad planteada mejoró el proceso de adquisición de conocimientos de los alumnos, ya que, aunque la búsqueda de información supuso un esfuerzo, los resultados finales demostraron que la actividad fue útil desde el punto de vista del aprendizaje, implicando a los alumnos en su propia formación. Ayudó a ampliar los conocimientos con un enfoque más práctico, que la tradicional clase magistral donde el profesor explica el tema.

Como aspecto de mejora, se ha planteado incluir en la base de datos y el programa etiquetas de alimentos que se encuentran en el mercado donde aparecen los aditivos en estudio. En algunas de las presentaciones realizadas por los alumnos presentaron fotografías de estas etiquetas, por lo que se sugirió incluirlas en la base de datos.

\section{REFERENCIAS}

Cabero-Almenara, J. (2010). Los retos de la integración de las TICs en los procesos educativos: Límites y posibilidades. Perspectiva Educacional, 49(1), 32-61-61. Retrieved from https://doi.org/10.4151/07189729-Vol.49-Iss.1-Art.3

Cabero-Almenara, J., \& Gutiérrez Castillo, J. (2015). La producción de materiales tic como desarrollo de las competencias del estudiante universitario. Aula de Encuentro: Revista de Investigación y Comunicación de Experiencias Educativas, 2(17), 5-32.

Cataldi, Z., \& Lage, F. J. (2012). TICs en educación: nuevas herramientas y nuevos paradigmas. VII Congreso de Tecnología En Educación y Educación En Tecnología, 11. Retrieved from http://hdl.handle.net/10915/18457 
González-Maura, V., \& González-Tirados, R. M. (2008). Competencias genéricas y formación profesional: un análisis desde la docencia universitaria. Revista Iberoamericana de Educación, 47(47), 185-209. Recuperado de http://www.rieoei.org/rie47a09.pdf

Ibáñez, F. C., Torre, P., \& Irigoyen, A. (2003). Aditivos alimentarios. Área de Nutrición y Bromatología, Universidad Pública de Navarra, Febrero, 1-10.

Llorente Cejudo, M. del C., Gutiérrez Porlán, I., \& Barroso Osuna, J. (2015). El papel del profesorado y el alumnado en los nuevos entornos tecnológicos. In Nuevos retos en tecnología educativa (pp. 217-238). Madrid: Síntesis.

Madrid, A. (1987). Manual de utilización de los aditivos en alimentos y bebidas. Madrid: Almansa.

Multon, J.-L. (1999). Aditivos y auxiliares de fabricación en las industrias agroalimentarias. Acribia.

Rodríguez Gómez, G., Ibarra Saiz, M. S., \& Cubero Ibáñez, J. (2018). Competencias básicas relacionadas con la evaluación. Un estudio sobre la percepción de los estudiantes universitarios. Educacion $X X 1,21(1), 181-208$. Retrieved from https://doi.org/10.5944/educXX1.14457 